\title{
MICROSYSTEM FOR ELECTROMECHANICAL MEASUREMENTS OF CARBON NANOFIBER LOADING AND FAILURE
}

\author{
J.J. Brown ${ }^{1}$, J.W. Suk ${ }^{2}$, G. Singh ${ }^{1}$, D.A. Dikin ${ }^{3}$, R.S. Ruoff ${ }^{2}$, and V.M. Bright ${ }^{1}$ \\ ${ }^{1}$ Department of Mechanical Engineering, University of Colorado, Boulder, CO, USA. \\ ${ }^{2}$ Department of Mechanical Engineering, University of Texas, Austin, TX, USA. \\ ${ }^{3}$ Department of Mechanical Engineering, Northwestern University, Evanston, IL, USA.
}

\begin{abstract}
A thermally actuated uniaxial testing stage for nanofiber materials has been designed and fabricated. Electrical separation of portions of the stage allows two-point electrical measurements simultaneously with mechanical testing. Using this stage, a carbon nanofiber was subjected to mechanical loading and simultaneous electrical impedance characterization, which provides a means to derive fiber resistance measurements when a fiber is mechanically coupled using highly resistive contacts.
\end{abstract}

\section{INTRODUCTION}

Electromechanical measurements on nanoscale fibers are of interest to enable integration of these materials into sensors and other microdevices. [1,2] Uniaxial testing is desirable in mechanical testing to ensure uniform loading throughout a fiber specimen [3,4], and to this end several devices have been developed to perform uniaxial mechanical testing on a nanofiber. [5-8] Furthermore, electrical coupling to a fiber specimen can allow thermomechanical characterization. The novelty of the device presented here lies in the ability to perform electrical measurement of a fiber specimen under mechanical loading, and mechanical loading of an electrically heated specimen.

\section{DESIGN}

\section{Actuation}

Stage actuation is realized by thermal expansion through Joule heating of a set of angled beams. Beams symmetrically connected to the stage ensure uniaxial motion and also serve as heat sinks. Using the actuator geometry and a plane strain condition, it was estimated through solid mechanics mathematical analysis that the thermal actuator would be capable of providing up to $400 \mu \mathrm{N}$ force. In order to avoid the specimen temperature changes caused by the actuator in $[5,6]$, the moving specimen stage was separated from the thermal actuator and mechanically anchored.

\section{Mechanical Measurements}

Tensile measurement is enabled by fixing a material specimen across a gap between the moving and fixed portions of the stage. The experiment reported here uses SEM observation as a direct means of measuring the carbon nanofiber strain. Microscopy requires the interpretation of micrographs in order to derive strain data, which can be a slow process. Sensors could provide a faster approach to acquisition of strain data, and to that end, several indirect strain measurement mechanisms were built into the reported device. These alternative devices can be seen at left in Figure 2, and as built in the center of Figure 1. These measurement approaches include a diffraction grating, piezoresistive beam bending, and electron emission or gas ionization. All these approaches require further characterization. The diffraction grating is designed to measure submicron stage displacement by examination of a reflected laser beam. The electron or ion sensor works by comparing an electron or ion current arriving at a fixed and a moving electrode.

For the piezoresistive beam measurements, electrical current is carried in two of the polysilicon anchoring beams. As the stage moves, the beams bend, modifying the electrical resistance of each beam. This concept has been explored in [9], but experimental results thus far have been inconclusive.

The successful implementation of one of the displacement sensors above can be used in combination with bending of a beam to derive a measured force as applied to the fiber, as in the approach taken in [8]. Direct force measurement has not yet been implemented in this design. An alternative approach to force measurement can be developed from measurements of the actuator input power and the stage displacement. The simulation shown in Figure 4 indicates that at any given applied voltage, there is a linear relation between force and displacement. This suggests that it may be possible to develop a map that translates electrical and stage displacement data into force measurements.

\section{Electrical Characterization}

Although two gold connections have been provided on the moving stage, they are electrically linked by the underlying polysilicon layer. Two separate electrical contacts were defined on the fixed side of the stage, effectively enabling a 3 point conductivity measurement. In practice, the difficulty of carbon nanofiber placement limited actual connections to only two: one on the moving stage, and one on the closest portion of the fixed stage. The fiber was mechanically clamped to the stage with amorphous carbon deposits created in an SEM, and these
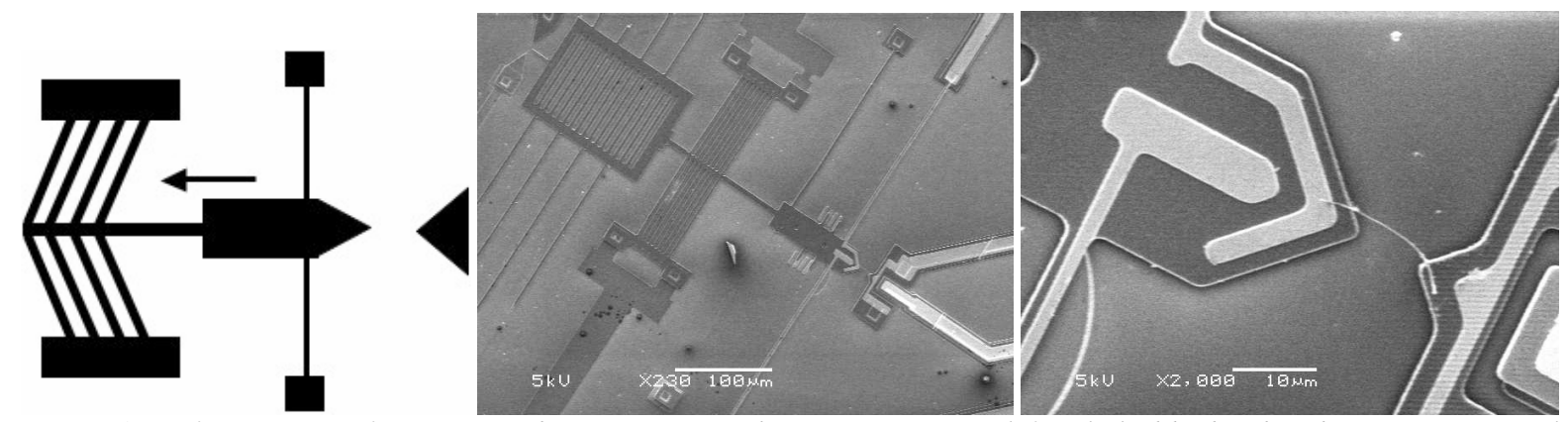

Figure 1. Left: Diagram of actuator and stage system. The moving stage at left is linked by bending beams to rectangular anchor points. A thermal actuator pulls the moving stage away from the fixed stage, at right. Center: SEM image of overall system. Right: Close-up of stage showing mounted carbon nanofiber, $120 \mathrm{~nm}$ diameter, before loading. 

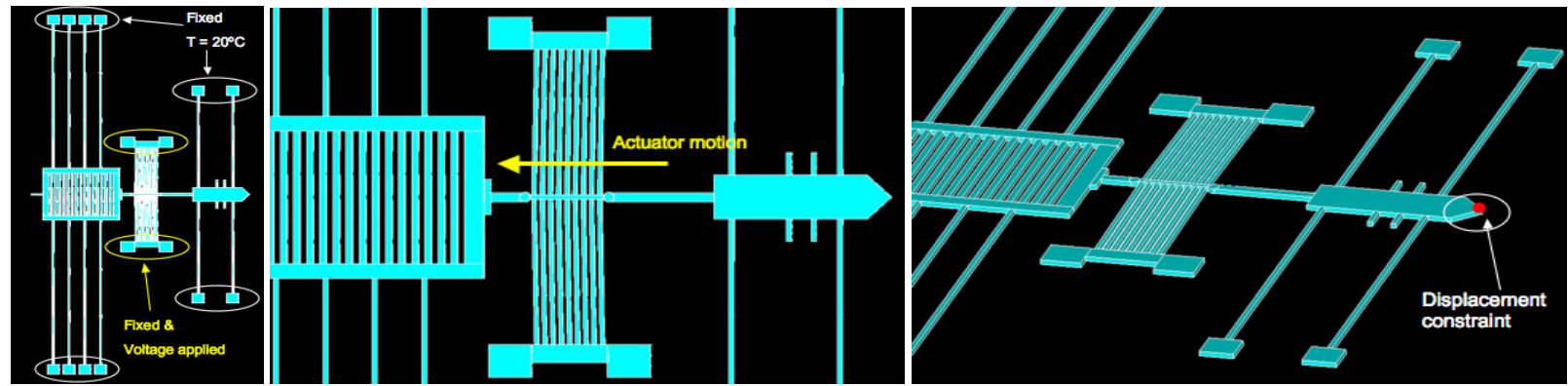

Figure 2: Layout for simulation. The moving stage is the pointed part at the right of the device, the thermal actuator is at the center, and the springs and large grating at the left were added to test several indirect means of displacement measurement.

amorphous carbon "welds" also served as the electrical connections. These amorphous carbon contacts present a high electrical resistivity, similar to the behavior of diamond-like carbon. Typically, only a very small amount of direct current can pass through these contacts. The structure of the contacts is one of a highly resistive material in a thin layer between two more conductive materials. In essence, these contacts are capacitors with lossy dielectrics. These capacitors lie in series with the fiber specimen, whose resistance is of interest in relation to strain behavior. Capacitors in a series circuit create a high-pass filter. At low frequencies and for DC currents, current must flow directly through the contacts and is therefore limited by the high resistance of the amorphous carbon contacts. As the frequency of an alternating current increases, the capacitors at the fiber contact points will begin to behave as if the contacts were shorted. Current at higher frequencies will capacitively couple across the contact points and the contact resistance will become irrelevant. A measurement of the AC circuit impedance will therefore be dominated by the resistance of the fiber and a reactance due to the contact capacitances.

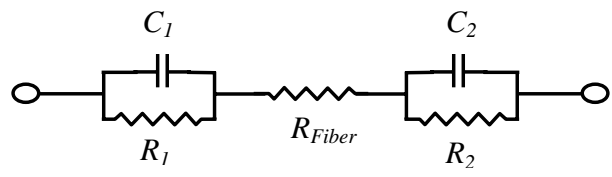

Figure 3. Circuit diagram for a fiber specimen with amorphous carbon contacts. Each contact has contact resistance $R_{1}$ and $R_{2}$, and contact capacitance $C_{1}$ and $C_{2}$. For fiber piezoresistive measurements, the resistance of the fiber $R_{\text {Fiber }}$ must be resolved.

Although the electrical connection to the moving stage is made on a bending beam, the piezoresistive change experienced by this beam is not significant for measurement of the fiber specimen. This is possible because a gold layer patterned on top of the connecting beam provides much greater conductivity, and the carbon specimen is connected only to this gold layer. Consequentially, current will be conducted primarily in the gold layer and only to a small extent in the polysilicon layer. The circuit probing the nanofiber resistance has some inherent resistance, and there may be some temperature-induced variation of the mounting stage resistance as the stage is warmed by the actuator. However, the small cross-section of the nanofiber implies that the nanofiber itself should have a large resistance that dominates other circuit resistances except for contact resistances.

The circuit from the LCR meter to the fiber specimen and back will have some inductance $L$ which may reduce the magnitude of the reactance observed. Inductive reactance $\mathrm{X}_{\mathrm{L}}$ is proportional to angular frequency $\omega$ and inductance, and capacitive reactance $X_{C}$ is inversely proportional to angular frequency and capacitance $\mathrm{C}$.

$$
\mathrm{X}_{\mathrm{L}}=\omega \mathrm{L} \quad \mathrm{X}_{\mathrm{C}}=-1 / \omega \mathrm{C}
$$

When both inductance and capacitance are small, and the AC frequency is kept relatively low (below the $\mathrm{MHz}$ range), the capacitive reactance will have a much larger value than the inductive reactance, and will therefore dominate.

\section{Electrical Connections}

Three electrical circuits are tied together by the moving stage. These are the thermal actuator circuit, the nanotube resistance measurement circuit, and, if desired, a displacement measurement circuit. If these circuits are not tied together at any other point, for instance if no more than one of the circuits is grounded, then the three circuits can operate independently.

Table 1. Polysilicon material properties used as simulation inputs. Data derived from references [10-12].

\begin{tabular}{|l|l|}
\hline \multicolumn{2}{|c|}{ Material Properties of Polysilicon } \\
\hline Thickness & $3.5 \mu \mathrm{m}$ \\
\hline Electrical Resistivity & $4.0 \times 10^{-4} \Omega-\mathrm{m}$ \\
\hline Thermal Conductivity & $150 \mathrm{~W} / \mathrm{m}-\mathrm{K}$ \\
\hline Poisson Ratio & 0.29 \\
\hline Modulus of Elasticity & $165 \mathrm{GPa}$ \\
\hline Coefficient of Thermal Expansion & $3 \times 10^{-6} / \mathrm{K}$ \\
\hline Yield Strength & $1.2 \mathrm{GPa}$ \\
\hline Density & $2.33 \times 10^{3} \mathrm{~kg} / \mathrm{m}^{3}$ \\
\hline
\end{tabular}

\section{SIMULATIONS}

A simulation was performed to define the thermomechanical behavior of the system across a range of applied input voltages. The simulation used the values in Table 1, the structure in Figure 2, and a tetrahedral coupled-field element, SOLID98, in ANSYS 10.0 software. The simulation did not include the temperature dependence of polysilicon electrical resistivity. In order to estimate the force applied from the stage to a mounted nanofiber, a displacement boundary condition was assigned to the moving stage. When no displacement constraint is assigned, corresponding to the absence of a test specimen, the simulation provides a parabolic curve of displacement versus voltage, similar in shape to the measured behavior, Figure 4. The discrepancy between the two curves can be explained in that the voltage was measured some distance away from the actuator.

At fixed voltages, with varying displacement constraints, the moving stage exhibits a linear reaction force versus displacement. The actuator effectively serves as a variably stiffened spring. Force simulation as shown in Figure 4 indicates the analytical estimate of $400 \mu \mathrm{N}$ to be a reasonable order of magnitude for a zero micron displacement constraint. The actuator experimentally demonstrated $>1.60 \mu \mathrm{m}$ displacement, also in good agreement with the values estimated in Figure 4. At $15 \mathrm{~V}$, the simulation predicts a stage temperature of $229^{\circ} \mathrm{C}$, significantly lower than the actuator temperature of $437^{\circ} \mathrm{C}$. 

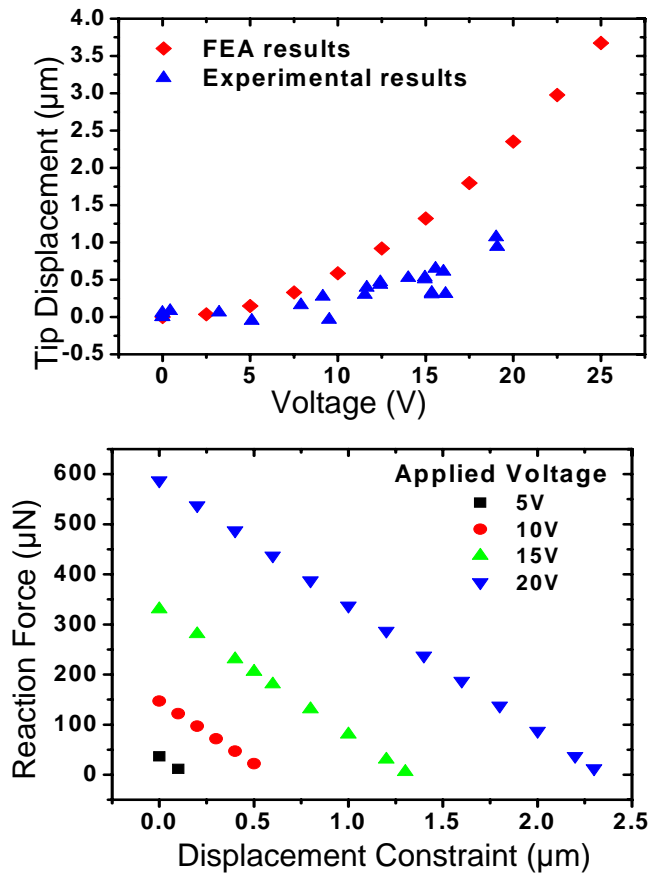

Figure 4. Simulation results. Top: Tip displacement versus voltage across the actuator for the freely moving stage. Bottom: Simulation output of the reaction force at the tip of the stage for different values of actuator voltage and constraints on the tip displacement.

\section{EXPERIMENTAL}

The device was fabricated from polysilicon with Au contacts using the PolyMUMPs service from MEMSCAP, Inc. The polysilicon layers were released using a 3 minute etch in $48 \%$ $\mathrm{HF}_{(a q)}$ followed by drying in a $\mathrm{CO}_{2}$ supercritical dryer.

Nanotube-core carbon nanofibers were synthesized using $\mathrm{Fe}$ thin film catalyst in a thermal chemical vapor deposition (CVD) reactor at $725^{\circ} \mathrm{C}$; they were subsequently ultrasonicated for 3 hours in a toluene solution and dried into the form of a mat. A 3-axis piezoelectric micro-manipulator (Kleindiek ${ }^{\mathrm{TM}}$ ) with a tungsten microscopy probe was used for separating an individual nanofiber from the mat using the procedures described in [13]. It was then transferred and glued on to the MEMS tensile stage by performing electron beam induced deposition (EBID) for 10 minutes with the JEOL JSM-6480LV scanning electron microscope in spot mode with $30 \mathrm{kV}$ accelerating voltage. Subsequently, the device substrate was bonded to a chip carrier, with wire-bonding for electrical connections, and this system was mounted in an SEM.

A DC power supply provided electrical current to the thermal actuator, and voltage on the power supply was varied to control the amount of power delivered to the thermal actuator. Voltage and current supplied to the actuators were measured using a Hewlett Packard 34401A multimeter. Using an Agilent 4263B LCR meter, the fiber resistance and reactance were observed as strain was varied. For these measurements, coaxial connectors were used to tie together the voltage and current lines on each side of the measured specimen. The stage displacement and nanofiber length were extracted from SEM images using ImageJ software.

\section{RESULTS AND DISCUSSION}

Before loading, DC resistance across the nanofiber was $>10$ $\mathrm{M} \Omega$. However, measurement at $100 \mathrm{kHz}$ found $5.0 \mathrm{k} \Omega$ resistance and $-55.5 \mathrm{k} \Omega$ reactance, indicating that contact resistance may dominate the DC measurement. The negative reactance indicates that the reactance is dominated by the capacitance. If the capacitance is inferred from the total reactance, an inline capacitance of $28.7 \mathrm{pF}$ is indicated by the measured reactance. This corresponds to a capacitance of $57.4 \mathrm{pF}$ at each contact if both contacts are assumed to have equal dimensions and permittivities. Measurement at $10 \mathrm{kHz}$ gave a value of $44 \mathrm{pF}$ for inline capacitance.

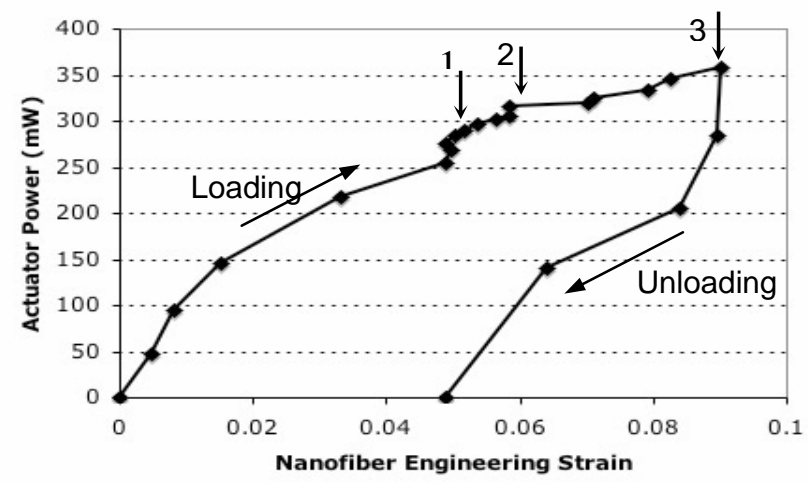

Figure 5. Strain determined from extension of the nanofiber from its length when completely unloaded. Following from the origin, the three marked inflection points indicate (1) where the fiber had straightened, (2) a point where the specimen resistance changed spontaneously under a constant strain, and (3) the point just after failure, seen at right in Fig. 7, where the fiber began to be unloaded.

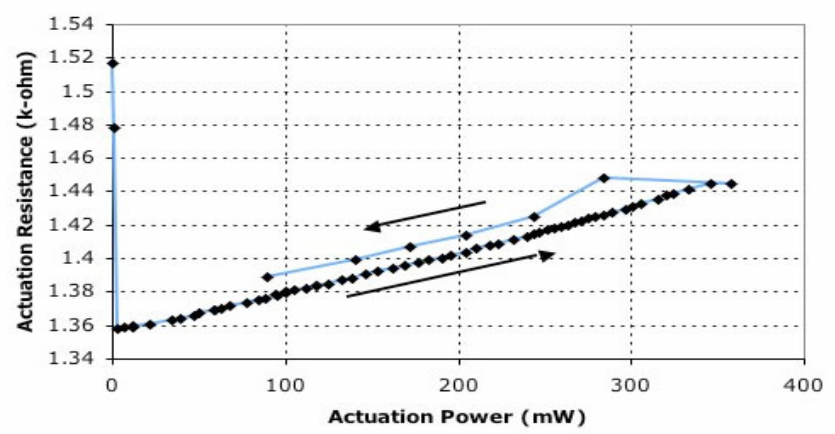

Figure 6. The polysilicon actuator shows a linear increase in electrical resistance with increasing input power, with a small hysteresis in resistivity upon reduction of the input power, possibly due to reduced loading from the nanofiber as the actuator power was reduced after the yielding of the fiber.

As the nanofiber was loaded, first it straightened and simultaneously elongated, until direct tensile load was applied to the length of the fiber. Failure eventually occurred in an outer layer of the fiber as seen in Figure 7. As the moving stage was allowed to return to its original position, the nanofiber buckled upon itself as seen in Figure 8, indicating that the failure seen in Figure 7 was not a complete fracture. In Figure 5 it can be seen that some strain was recovered as the fiber was unloaded, indicating that the observed nanofiber failure was a plastic deformation. Another interpretation of this data could be that the fiber moved out of the plane of observation as the stage was allowed to retract.

In Figure 6, it can be seen that the actuator resistance increases with the input power and therefore with the temperature of the actuator. This indicates that the thermomechanical simulation of the actuator could be more accurate if it were to include temperature-dependent electrical resistivity. However, the actuator 

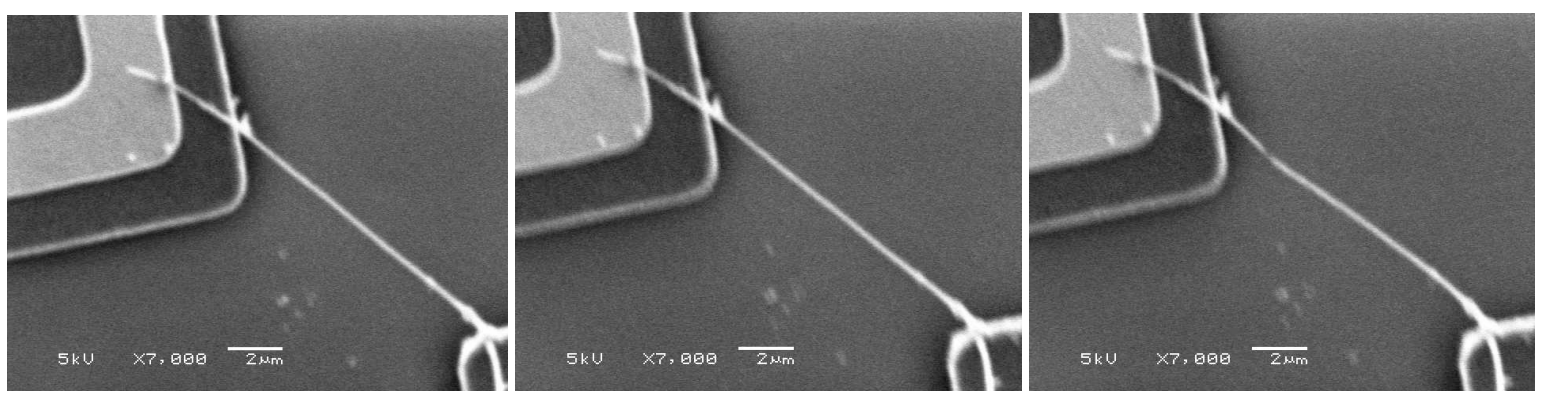

Figure 7: Tensile observation. Left: Slack removed from nanofiber before significant loading, $R=5.01 \mathrm{k} \Omega$ (measured at $100 \mathrm{kHz}$ ). Center: Nanofiber just before failure, $\varepsilon=5.3 \%$. $R=4.98 \mathrm{k} \Omega$. Right: Failure of an outer layer. $R=4.98 \mathrm{k} \Omega$. Reactances ranged from $X=-54.7 \mathrm{k} \Omega$ to $-57.0 \mathrm{k} \Omega$. No significant trends in resistance or reactance were observed for this sample during the tensile test.

exhibits a hysteresis in resistance as the actuator is unloaded. A typical temperature-dependent resistance simulation would not account for this behavior. Some possible explanations of this hysteresis include a piezoresistive effect on the loaded actuators, or microstructural changes due to the elevated temperatures.

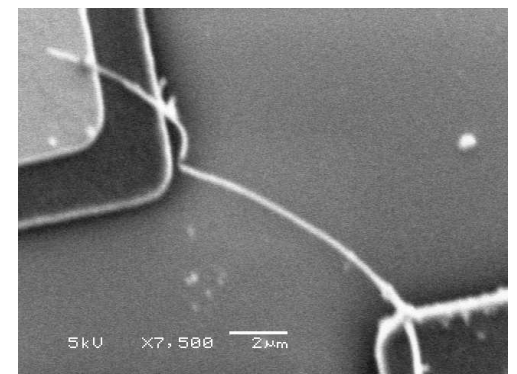

Figure 8. Image of the nanofiber after the moving stage has been allowed to return to its original position.

\section{CONCLUSIONS}

A novel stage for electromechanical testing of micro and nanoscale fibers has been designed, simulated, and fabricated. Further thermal design is required to minimize fiber resistance measurement error due to stage temperature deviations caused by the thermal actuators.

For carbon nanofiber testing, specimens were placed using a micromanipulator and bonded using amorphous carbon deposited in an SEM. Specimen manipulation and placement, and derivation of strain data from micrographs are key limiting steps to data collection. The development of faster approaches to displacement measurement and to nanofiber placement is needed to improve the quality of mechanical characterization data of nanoscale fibers.

The use of AC two-point impedance measurements has been demonstrated as an approach to bypassing the high contact resistance from mechanical welds to tensile specimens. Impedance measurements may additionally provide an estimation of the capacitance of these welds.

\section{ACKNOWLEDGEMENTS}

This research was supported by DARPA Award \# HR0011-061-0048 and the DARPA Focus Center for Integrated Micro/NanoMechanical Transducers (iMINT Center) at the University of Colorado, Boulder. This material is based upon work supported under a National Science Foundation Graduate Research Fellowship, which supports J.J. Brown. The authors wish to thank NIST-Boulder and Paul Rice at the University of Colorado for help with the nanotubes and the micromanipulator.

\section{REFERENCES}

[1] A. Jungen, C. Stampfer, J. Hoetzel, V.M. Bright, C. Hierold, "Process integration of carbon nanotubes into microelectromechanical systems”, Sensors \& Actuators A, 130, 588 (2006).

[2] C. Stampfer, A. Jungen, and C. Hierold, "Fabrication of discrete nanoscaled force sensors based on single-walled carbon nanotubes”, IEEE Sensors Journal, 6, 613 (2006).

[3] M.F. Yu, O. Lourie, M.J. Dyer, K. Moloni, T.F. Kelly, and R.S. Ruoff, "Strength and Breaking Mechanism of Multiwalled Carbon Nanotubes Under Tensile Load”, Science, 287, 637 (2000).

[4] M.-F. Yu, B.S. Files, S. Arepalli, and R.S. Ruoff, “Tensile Loading of Ropes of Single Wall Carbon Nanotubes and their Mechanical Properties”, Phys. Rev. Letters, 84, 5552 (2000).

[5] S. Lu, D.A. Dikin, S. Zhang, F.T. Fisher, J. Lee, and R.S. Ruoff, "Realization of nanoscale resolution with a micromachined thermally actuated testing stage”, Rev. Sci. Inst., 75, 2154 (2004).

[6] S. Lu, Z. Guo, W. Ding, and R.S. Ruoff, “Analysis of a microelectromechanical system testing stage for tensile loading of nanostructures”, Rev. Sci. Inst., 77, 056103 (2006).

[7] M. Kiuchi, S. Matsui, and Y. Isono, "Mechanical Characteristics of FIB Deposited Carbon Nanowires Using an Electrostatic Actuated Nano Tensile Testing Device”, Journal of Microelectromechanical Systems, 16, 191 (2007).

[8] Y. Zhu, A. Corigliano, and H.D. Espinosa, “A thermal actuator for nanoscale in situ microscopy testing: design and characterization”, Journal of Micromechanics and Microengineering”, 16, 242 (2006).

[9] T.L. Waterfall, G.K. Johns, R.K. Messenger, B.D. Jensen, T.W. McLain, and L.L. Howell, "Observations of piezoresistivity for polysilicon in bending that are unexplained by linear models", Sensors \& Actuators A, 141, 610 (2008).

[10] T. Moulton and G.K. Ananthasuresh, "Micromechanical devices with embedded electro-thermal-compliant actuation”, Sensors and Actuators, A, 90, 38 (2001).

[11] C.-S. Oh and W.N. Sharpe, Jr., "Techniques for measuring thermal expansion and creep of polysilicon”, Sensors \& Actuators, A: Physical, 112, 66 (2004).

[12] W.N. Sharpe, Jr., B. Yuan, R. Vaidyanathan, and R.L. Edwards, "Measurements of Young's modulus, Poisson's ratio, and tensile strength of polysilicon", Proceedings of IEEE MEMS '97, (1997), pp. 424-429.

[13] G. Singh, P. Rice, K.E. Hurst, J.H. Lehman, and R.L. Mahajan, "Laser-induced exfoliation of amorphous carbon layer on an individual multiwall carbon nanotube”, Applied Physics Letters, 91, 033101 (2007). 